Schubert, Karsten (2020): Queere und schwule Theorie. In: Clemens Kammler, Rolf Parr und Ulrich Johannes Schneider (Hg.): Foucault-Handbuch. Leben - Werk - Wirkung. 2., aktualisierte und erweiterte Auflage 2020. Stuttgart: J.B. Metzler, Part of Springer Nature - Springer-Verlag GmbH; J.B. Metzler, S. 503-509. https://doi.org/10.1007/978-3-476-057174_92

Manuskript. Wenn Sie keinen Zugang zur Verlagsversion haben, wenden Sie sich gerne per Email an mich.

\title{
Foucault-Rezeption
}

\section{Queere und schwule Theorie}

Foucault bildet eine zentrale Grundlage der queeren und schwulen Theorie, die sich seit den späten 1980er Jahren insbesondere in den USA entwickelt hat. Seine Macht- und Subjekttheorie ist die Basis für eine nicht- essentialistische Analyse von Sexualität und für die Kritik ihrer normierenden Wirkung, die Foucault selbst in Der Wille zum Wissen (1983, frz. 1976) begonnen hat und die das Kerngeschäft der Queertheorie ist. Während Foucault als Grundlage der Queertheorie insgesamt rezipiert wird, gibt es eine spezifisch schwule Rezeption von Foucault, die an seine vielfältigen Äußerungen zur schwulen Politik anschließt und dessen Machtanalyse und seine späteren Arbeiten zur Ästhetik der Existenz mobilisiert, um damit das schwule Leben vor, während und nach der AIDSKrise zu analysieren.

\section{Foucault als queerer und schwuler Theoretiker}

Sechs Monographien beschäftigen sich mit dem Verhältnis von Foucault und der Queertheorie und erläutern die fundamentale Bedeutung seines Denkens für die queere Gesellschaftskritik. David Halperins Saint Foucault von 1995 sticht heraus, weil Halperin Foucaults Denken mit großer Detailschärfe und in tiefer Kenntnis der allgemeinen Foucault-Rezeption aus einer queeren und schwulen Perspektive entfaltet. Die nunmehr 25 Jahre alte Monographie ist zum Referenzpunkt weiterer Beiträge zu Foucault und Queer geworden und hat nichts an ihrer Aktualität eingebüßt. Im Gegensatz zum heterosexuellen Blick insbesondere der sozialphilosophischen Kritiker*innen an Foucaults Methode und an seinem Interesse an antiker Ethik wird bei Halperin Foucaults spezifisch schwule Perspektive intelligibel, ohne dass Foucault homophob auf sein 
Schwulsein reduziert würde - wie es Halperin einigen heterosexuellen Rezipienten vorwirft. Das ärgerliche Unverständnis und die Fehlrezeption der frühen sozialphilosophischen Foucault-Kritiker*innen, wie Charles Taylor, Edward Said, Jürgen Habermas und Nancy Fraser, das in der Fachdebatte mittlerweile korrigiert ist, wird durch die neue Dimension - die heterosexuelle Perspektive der Debattierenden - besser erklärt als aus rein sozialphilosophischen Missverständnissen. Im Folgenden geht es um zwei zentrale Aspekte der Studie: Wie Foucaults Machttheorie eine neue Analyse und Kritik von Sexualität ermöglicht und wie seine Konzepte der Ethik, der Ästhetik der Existenz und der genealogischen Kritik die transformative und kreative Neuschaffung von schwulen und queeren Lebensweisen beschreiben, die Widerstand gegen die Anpassung an heteronormative Lebensstile leisten. Beide Aspekte - der machtanalytisch-kritische und der transformativkreative - tauchen in fast allen queertheoretischen Texten auf.

Halperin geht von der Beobachtung aus, dass Foucaults Der Wille zum Wissen der zentrale theoretische Text der AIDS-Aktivist*innen war, insbesondere von ACT-UP in den späten 1980er und frühen 90er Jahren, die queere Kritik avant la lettre betrieben (17). Er fragt:»What did gay activists see in Foucault, and specifically in The History of Sexuality, Volume I, that his straight-liberal critics missed, and why? « (26). Halperins These ist, dass Foucaults Machtund Subjekttheorie besonders geeignet ist, um moderne Homophobie zu analysieren. Foucault bot erstens die Theorie zur AIDS-Krise: Dass Sexualität ein besonderer Knotenpunkt der Macht ist, dass die Autorität von Expert*innenwissen sie zusammen mit Machttechnologien und biopolitischen Regierungsrationalitäten reglementiert und dadurch der Körper zum Ort politischer Kämpfe wird - das ist die Realität des AIDS-Aktivismus (27 f.). Zweitens sei Foucaults These, dass Freiheit nicht außerhalb der Macht liegt, als eine Befreiung von ihr, sondern nur als widerständige und kritische Praxis gedacht werden kann, eine Beschreibung der schwulen Praxis des Camps, also der widerständigen Umdeutung und parodierenden Aneignung von existierender heterosexueller Kultur wie beispielsweise beim Drag (29). Genau ein solcher Akt des Widerstandes und nicht der Befreiung ist drittens das Coming-Out, denn die repressive Macht der homophoben Normierung wird dadurch nicht aufgehoben, sondern man ändert seine Position im strategischen Umgang mit ihr (29 f.). Nichts macht Foucaults Einsicht in die Allgegenwart der Macht deutlicher als das Closet und das Coming-Out. Zentral ist nach Halperin viertens, dass Foucault ein grundsätzlich neues Verständnis der Sexualität anbietet, das sie nicht als eine essentielle oder biologische Eigenschaft, sondern als ein Produkt und Konstrukt von vermachteten Diskursen bestimmt. Der Begriff der Macht-Wissens-Komplexe macht eine Alternative dazu möglich, der 
Homophobie durch neue Wahrheiten über den Sex zu begegnen, wie frühere Theorien zur Befreiung der Sexualität es versucht haben - eine Strategie, die wegen der Widersprüchlichkeit homophober Diskurse nach Halperin aussichtslos ist. Mit Foucaults Theorie kann der Fokus vielmehr auf die exponierende Kritik derSexualitätsdiskurse und ihrer widersprüchlichen Konstruktionen gerichtet werden. Auf dieser Grundlage - und gerade wegen ihrer marginalisierten Position im Diskurs - können aus den shomosexuellen Objekten des Expert*innenwissens schwule politische Subjekte werden, die die wissenschaftliche Wahrheitsproduktion über ihre »Spezies«als Machtechnologie ablehnen: »The homosexual subject can now claim an identity without an essence« (42, 60 f.).

>Homosexualität als Potential zur Entwicklung von kreativen und transformativen Lebensweisen und nicht als substantielle Identität zu betrachten, ist ein direktes Ergebnis dieser machtanalytischen und genealogischen Kritik der Sexualität. Foucault äußert sich entsprechend in mehreren Veröffentlichungen vor allem in schwulen Magazinen:»Wir müssen also darauf hinarbeiten, homosexuell zu werden und dürfen uns nicht darauf versteifen, dass wir es schon sind.«(Foucault1984,85)DamitbringtFoucaultden transformativen Gehalt des heutigen Begriffs squeer auf den Punkt und hätte heute entsprechend squeer anstatt >homosexuelk geschrieben (Halperin 1995, 66-68). Dabei ist»[s]elf-invention [...] not a luxury or a pastime forlesbians and gay men: it is a necessity (81), eine Notwendigkeit, die aus der homophoben Normierung und ihren eingeschränkten Formen des Zusammenlebens, wie der Ehe, folgt. Foucault reflektiert die Möglichkeiten solcher schwulen Selbsterfindung durch seine Analysen der antiken Ethik der Existenz (68 ff.). Dabei geht es nicht darum, die Wahrheit seines Selbstzu entdecken, sondern sich in Relation zu anderen neu zu erfinden. Halperin erläutert, wie für Foucault die schwule Kultur, insbesondere die BDSM-Szene, in die er in San Francisco eintauchte, eine solche zeitgenössische Ästhetik der Existenz ist. Zentral ist dabei, dass es sich um eine freie, gemeinschaftliche und nichtnormalisierende Praxis der Selbsterfindung handelt. Dass die antike Askese sich als selbstauferlegte Einschränkung der Sexualität verstand, die auf den ersten Blick weit entfernt von der Entfaltung der Lüste in der BDSM-Kultur ist, spielt für diese systematische Verwandtschaft der Ästhetiken keine Rolle (77, 85-90): »Foucault goes on to hypothesize that 〉Gide in Greece would have been an austere philosopher; by the same token, one might speculate that Seneca in San Francisco would have been a gay leatherman - and a butch bottom, at that « (103). Doch subkulturelle Praktiken und insbesondere kritische Theorien tendieren auch dann zur Normalisierung, wenn sie inhaltlich dagegen gerichtet sind. Kritisch attestiert Halperin dies schon 1995 der sich 
institutionalisierenden Queertheorie und wiederholt diese Diagnose später (Halperin 2003). Genau gegen solche Institutionalisierungen hätte Foucault seine nicht-normativen und nicht-kodifizierenden Analysen von Normen und Freiheitspotentialen ausgerichtet (Halperin 1995, 133). Genützt hat es, wie im zweiten Abschnitt deutlich wird, bezüglich der Queertheorie wenig, die zumindest in den USA längst eine klassische akademische Disziplin geworden ist. Allerdings liegt es nahe, im Anschluss an Foucault und gegen Halperin, diese Institutionalisierung des queeren Denkens in der Akademie als ein Mittel im strategischen Kampf gegen homophobe Diskurse zu beschreiben und als solche zu begrüßen.

Lynne Huffers Mad for Foucault (2010) argumentiert, dass nicht nur Der Wille zum Wissen, sondern insbesondere Wahnsinn und Gesellschaft (1973, frz. 1961), das im Englischen erst seit 2006 in kompletter Länge vorliegt, für die Queertheorie von entscheidender Bedeutung ist. Huffer zeigt, wie `Queerness in der Moderne mit Unvernunft verbunden wurde. Mike Laufenberg bietet in Sexualität und Biomacht (2014) eine Interpretation von Foucaults Biopolitiktheorie in Hinblick auf queere Sexualität. Laufenberg kritisiert dort auch die (heteronormative) Ausblendung der Sexualität in den etablierten biopolitischen Ansätzen. Das kleine Büchlein Foucault and Queer Theory (Spargo 1999) empfiehlt sich als Einleitung in die queere Politik und Theorie, Foucaults Werk und den Zusammenhang zwischen beiden. Didier Eribons Betrachtungen zur Schwulenfrage (2019), in Frankreich 1999 veröffentlicht, entwickelt nach Foucault eine Theorie der Beleidigung und Stigmatisierung, wobei der dritte Teil des Buches der dafür nötigen Re-Interpretation von Foucaults Werk gewidmet ist. Ladelle McWorthers Bodies and Pleasures (1999) ist eine persönliche Foucault-Lektüre aus lesbischer Perspektive, die den transformativen Effekt von Foucaults Kritik bezeugt. Zentral für die queere und feministische Rezeption Foucaults ist Judith Butler (s. Kap. 37).

\section{Normierung und Historisierung}

Gayle Rubins »Thinking Sex« (2011) von 1986, eines der Gründungsdokumente der Queertheorie, ist tief von Foucaults Machtanalyse geprägt. Rubin sieht die zunehmende Repression und Pathologisierung von sexueller Vielfalt in den USA der 1980er als einen Wendepunkt in der Politik der Sexualität - wie schon vorher die 1880er und die 1950er Jahre -, zu deren Analyse eine neue radikale Theorie der Sexualität nötig ist (138-144). Sie erläutert sechs Annahmen über Sexualität in Wissenschaft, Gesellschaft und Kultur, die der Entwicklung einer solchen Theorie entgegenstehen und deshalb überwunden 
werden müssen. Die erste und wichtigste Annahme ist sexueller Essentialismus, also die Annahme, dass Sexualität eine natürliche, vorsoziale und historisch invariable Kraft sei, die nur auf Biologisches zurückführbar sei (146). Neue Forschung setzt dagegen eine konstruktivistische Auffassung von Sexualität als abhängig von sozialen Normen, Machtverhältnissen und historischen Denkweisen: »Michel Foucault's The History of Sexuality has been the most influential and emblematic text of the new scholarship on sex" (146). Der konstruktivistischen Perspektive im Generellen und Foucault im Speziellen wurde vorgeworfen, Repression von Sexualität nicht denken zu können. Rubin stellt klar, dass Foucault nie die Existenz von sexueller Repression bestritten hat, sondern sie in eine größere gesellschaftliche Dynamik einordnet. Das ist ein entscheidender Hinweis angesichts der oft verkürzten Rezeption von Foucaults Kritik der Repressionshypothese. Foucault unternimmt also, was auch die Aufgabe der Queertheorie ist: die Analyse von sexueller Repression, ohne dabei auf essentialistische Modelle zurückzugreifen, wie der Freudomarxismus (147 f.).

Die fünf weiteren geläufigen Annahmen über Sexualität, die von einer radikalen Theorie überwunden werden sollen, sind nach Rubin (148-154): Eine grundsätzlich negative Bewertung von Sexualität(1), der aber dennoch enorme kulturelle Wichtigkeit zugemessen wird (2) und die in einem strikten Hierarchiesystem organisiert ist, ähnlich wie rassistische Ideologie (3). Dabei steht monogamer, reproduktiver Sex in der Ehe an der Spitze und kommerzieller, promisker, homosexueller Sex ganz unten. Folglich gibt es eine Angst vor dem kompletten Einbruch der Moral durch kleine Abweichungen von der sguten Sexualität(4) und eine Ablehnung von sexueller Vielfalt (5). Dieses Denken muss überwunden werden, um ein demokratisches Modell der Sexualität zu entwickeln, bei dem Konsens und Lust der Partner*innen im Mittelpunkt stehen (154).

Die epistemologischen Widersprüche des westlichen homophoben Diskurses erläutert Eve Sedgwick in ihrem Klassiker The Epistemology of the Closet (1990) und vertieft damit Foucaults Analysen des Sexualitätsdispositivs (3). Sie beschreibt die Wirkung der Unterscheidung von Homo- und Heterosexualität, die sich in der dritten Hälfte des 19. Jh.s in Deutschland ausgebildet hat und eine erklärungsbedürftige Dominanz entfalten konnte. »What was new from the turn of the century was the world-mapping by which every given person, just as he or she was necessarily assignable to a male or a female gender, was now considered necessarily assignable as well to a homo- or a hetero-sexuality (2). Diese Binarität ist voller Widersprüche, Unklarheiten und Halbwissen. Nach Sedgwick ist es genau diese Widersprüchlichkeit, die homophobe Diskurse so mächtig werden lässt. Das ist mit dem Titel angezeigt: An assumption underlying the book is that the relations of the closet - the relations of the known and the unknown, the explicit and the inexplicit around 
homo/heterosexual definition have the potential for being peculiarly revealing, in fact, about speech acts more generally« (3). Sedgwick zeigt im Anschluss an Foucault, wieSchweigen, Unwissenheit und Ignoranz Machtstrategien sind, beispielsweise bei der Zurückweisung des Rechts auf konsensualen Analsex des U. S. Supreme Court als scherzhaft und lächerlich (6 f.). Wie Halperin spricht sich deshalb auch Sedgwick nicht für eine Strategie der Etablierung einer neuen Wahrheit über den Sex zur Korrektur des Unwissens aus, sondern dafür, die Widersprüche im Diskurs zu analysieren und machtkritisch zu multiplizieren (8), was einer Methode der Dekonstruktion gleichkommt: »A deconstructive understanding of these binarisms [hetero/homo und natürlich/unnatürlich; K. S.] makes it possible to identify them as sites that are peculiarly densely charged with lasting potentials for powerful manipulation - through precisely the mechanisms of self-contradictory definition « (10, Herv. im Orig.). Durch diese Methode der dekonstruktivistischen Machtkritik entwickelt Sedwick beispielsweise eine kritische Position zur Debatte um Essentialismus und Konstruktivismus, die sie für obsolet hält, »because I am very dubious about the ability of even the most scrupulously gay-affirmative thinkers to divorce these terms, especially as they relate to the question of ontogeny, from the essentially gay-genocidal nexuses of thought through which they have developed (40). Diese Ablehnung der Frage zur Entstehung der`Homosexualität mit dem Hinweis, dass nicht nur die essentialistische, sondern auch eine sozialkonstruktivistische Auffassung gefährlich sein kann, ist brandaktuell, wie die rechte homophobe Politik der späten 2010er Jahre zeigt: Sie basiert implizit auf sozialkonstruktivistischen Argumenten, wenn argumentiert wird, neue Bildungspläne und queere >Propagandar würden die Kinder vom rechten Weg der Heteronorm abringen.

Es wäre aber verfehlt, diesen Hinweis auf den homophoben Hintergrund der Frage nach derErklärung von >Homosexualität als die queertheoretische Konklusion der Debatte um Essentialismus und Konstruktivismus zu verstehen. Einerseits, weil die emanzipative Wirkung einer konstruktivistischen Betrachtung die Gefahr einer reaktionären Umkehrung überwiegt. Andererseits aber, weil es in dieser wissenschaftlichen Debatte einen tatsächlichen Erkenntnisfortschritt gegeben hat, der zeigt, dass der Konstruktivismus plausibel ist. Wie genau eine solche konstruktivistische Analyse der männlichen ১Homosexualität‘ unternommen werden kann, zeigt Halperins »How to do the history of gay male homosexuality (2000; s. auch Spector et al. 2012). Er fragt »how exactly—in what terms, by virtue of what temporality, in which of its dimensions or aspectssexuality does have a history (87). Nach Foucault entwickelt er dafür einen genealogischen Konstruktivismus, der vom zeitgenössischen Begriff der Homosexualität ausgeht, weil in ihm die Entwicklungslinien der Vergangenheit 
kulminieren. Die Inkonsistenz des Begriffs wird dadurch nicht zum Problem, sondern zum Einstiegspunkt der genealogischen Analyse der diskontinuierlichen und widersprüchlichen Einzelaspekte (90), die im Begriff der Homosexualität verschmelzen, was dievon Sedgwick konstatierte Inkohärenz des Begriffs erklärt. In einer aufschlussreichen historischen Kulturanalyse beschreibt Halperin vier vormoderne und "prähomosexuelle« Kategorien, die Gender gegenüber Sexualität priorisieren, was sich dann im Begriff der Homosexualität umkehrt. (1) Feminisiertheit, (2) Päderastie bzw. aktive Sodomie, die auf klaren Geschlechterrollen (aktiv und passiv) beruht und nicht als sexuelle Orientierung gedacht wird, (3) Freundschaft zum Ausdruck gleichrangiger Beziehungen und (4) Inversion, als krankhafte totale Aufgabe der Männlichkeit. Der Begriff der Homosexualität konstruiert erstmals eine sexuelle Orientierung und kombiniert die Elemente psychologische Kondition, gleichgeschlechtliche Objektwahl und abweichendes Sozialverhalten und umfasst damit zum ersten Mal jedes gleichgeschlechtliche Begehren und Verhalten, unabhängig von Hierarchie und Gender. >Homosexualität ist im Gegensatz zu den prähomosexuellen Konzepten egalitärer, was neue gleichberechtigte homosexueller Beziehungen ermöglicht. Andererseits führt die Schaffung der >Homosexuellen als Spezies auch dazu, dass (heterosexuelle) Männer sich in der Gefahr sehen, für homosexuell gehalten zu werden, was ein Grund für die enorme Zunahme der Homophobie im 20. Jh. ist (122).

\section{AIDS-Krise und die These der Antisozialität schwuler Sexualität}

Viele Beiträge der queeren und schwulen Theorie sind Analysen der neuen Repression und Stigmatisierung während der AIDS-Krise. Leo Bersanis Artikel »Is the Rectum a Grave? «(1987) ist eine schonungslose Kritik normierender Effekte der liberalen und bürgerlichen Antworten auf die AIDS-Krise und entwickelt dabei eine grundsätzliche These zum negativen und anti-sozialen Charaktervon Sex, die viel Resonanz in der Queertheorie gefunden hat: die "Antisocial Thesis«. Bersani kritisiert, wie schwuler Sex von vielen Aktivist*innen frei, konsensual und divers dargestellt wird, um rechten Vernichtungsphantasien zu begegnen, indem sie zeigen: Wir sind gute bürgerliche Subjekte. Dabei räumt er mit einigen optimistischen Gemeinplätzen auf, wie, dass Cruising und schwule Saunen demokratische Orte sozialer Egalität seien (206), oder dass die schwule Verehrung und Reproduktion maskuliner Stile ein Mittel zur politischen Subversion und Parodie sexy sein könne (208). Er hält dagegen, dass Schwulsein nicht per se politisch radikal ist (205). Weiter noch: Das Begehren nach Männern, das trotz der sozialen Konstruktion von Geschlechternormen immer das Begehren nach den jeweils 
gültigen - also nach wie vor machohaften, heute würde man sagen toxischmaskulinen-Männlichkeitsformen ist, führt sogar zu einer grundsätzlichen Internalisierung und Identifizierung mit homophober Maskulinität (209). Schwule Sexualität hat aber trotzdem das Potential, Maskulinismus zu sprengen. Das funktioniert aber nicht in einem liberalen Modell, sondern nur durch die Untersuchung der Negativität des Sex, wofür Bersani von der Beobachtung ausgeht, dass Schwule die heterosexuelle Männlichkeit nicht durch Parodie zersetzen, »but rather because, from within their nearly mad identification with it, they never cease to feel the appeal of its being violated « (209; Herv. im Orig.).

In einer kreativen Aneignung von radikalfeministischer Theorie entwickelt Bersani die These, dass Sex inhärent gewaltvoll, männliche Penetration vernichtend und penetriert zu werden die totale Aufgabe von Macht sei (213). Bersani analysiert, dass es ein quer durch die politischen Lager geteiltes Projekt der "redemptive reinvention of sex" (215) als gewaltfrei, konsensual, sozial und liebend gebe, das in seiner Normativität eine pastorale Funktion hat. Diese humanistische Normativität des Sexes sieht Bersani nicht nur bei sexpositiven und sexnegativen Feminist*innen, sondern auch in der schwulen Theorie am Werk, inklusive Foucaults Spekulationen über den Wert der »Lüste«. Bersani radikalisiert also Foucaults Kritik, indem er die auch bei ihm vorhandenen Elemente des normalisierenden Denkens aufdeckt und in einen größeren Diskurs einordnet. Gegen das pastoral-bürgerliche Modell behauptet Bersani »the inestimable value of sex as - at least in certain of its ineradicable aspects - anticommunal, antiegalitarian, antinurturing, antiloving“ (215). Der Wert des Sexes liegt nach Bersani gerade in der Ausübung und im tatsächlichen Verlust von Macht (216). Bürgerlicher Phallozentrismus ist dagegen die Verneinung des Wertes der Machtlosigkeit, womit Bersani die »radical disintegration and humiliation of the self« meint (217). Sexualität kann das stabile, bürgerliche Selbst zersetzen und transformieren, das die Basis für (sexistische) Herrschaftsverhältnisse ist (218). Gegen die pastorale Reinigung der Sexualität, die schwule Promiskuität, insbesondere passiven Analsex, entweder verurteilt oder in eine bürgerliche Diversitätserzählung einordnet, hält Bersani entsprechend fest:»Butif the rectum is the grave in which the masculine ideal (an ideal shared - differently - by men and women) of proud subjectivity is buried, then it should be celebrated for its very potential for death« (222). Die AntisocialThesis wurde kontrovers diskutiert, wobei Foucault den geteilten Hintergrund der Debatte und der darin formulierten Kritik an der Integration des queeren Sexes in die bürgerliche Gesellschaft bildet (Edelman 2004; Berlant/Edelman 2014; Muñoz 2009; Caserio et al. 2006). 


\section{Homonormativität und Homonationalismus}

Der Kampf um die rechtliche Gleichstellung von Schwulen und Lesben hat seit den 2000er Jahren in westlichen Demokratien enorme Fortschritte gemacht (Schubert 2013; Trott 2016). Die neuere queere Theorie in Foucault'scher Tradition setzte die Kritik an der normalisierenden und ausschließenden Funktion der damit einhergehenden Verbürgerlichung fort und hat dafür zwei zentrale Konzepte entwickelt: Homonormativität und Homonationalismus. Während der Begriff >Homonormativität von Lisa Duggan (2002-2003) geprägt wurde, finden sich zentrale Gedanken systematischer in Laurent Berlants und Michael Warners Klassiker »Sex in Public» (1998). Sie kritisieren die heteround homonormative Verschiebung von Sexualität in private Intimität und zeigen dagegen, dass gegenöffentliche Orte der Sexkultur, wie Clubs oder Bars, die Basis für queere Emanzipation sind. Thre Kritik an den bürgerlichen Schwulen, die nur heiraten wollen, aber sich von queerer (Sex-)Kultur distanzieren, ist angesichts des heutigen Rechtspopulismus aktueller denn je: »Respectable gays like to think that they owe nothing to the sexual subculture they think of as sleazy. But their success, their way of living, their political rights, and their very identities would never have been possible but for the existence of the public sexual culture they now despise. Extinguish it, and almost all out gay or queer culture will wither on the vine. No one knows this connection better than the right (563; vgl. Warner 1993, 2000; Halperin/Traub 2009; Halperin 2012). Homonationalismus wird als ein spezifischer, aber nicht notweniger Aspekt von Homonormativität verstanden. Der Begriff wurde von Jaspir Puar geprägt (2007) und dient der Analyse und Kritik der Anschlussfähigkeit von bürgerlichen Schwulen und Lesben an nationalistische und konservative Politikprojekte. Dies betrifft vor allem die Rechtfertigung anti-muslimischer Politik und nationaler Abschottung durch die rassistische Konstruktion von grundsätzlich homophoben Muslimen, vor deren Einwanderung westliche Schwule und Lesben geschützt werden müssten.

Die Kritik an Homo- und Heteronormativität in der queeren Theorie ist heute intersektional, und Foucaults Macht- und Subjekttheorie bildet einen kontinuierlichen Bezugspunkt dieser Ausdifferenzierung. Dabei hat sich die Thematisierung von trans* (Halberstam 2018; Preciado 2013) mittlerweile zum eigenständigen Forschungsfeld der Trans*-Studies entwickelt (Keegan 2018). Die Intersektion von Queerness und Race ist Gegenstand vieler aufschlussreicher Studien (Scott 2010; Nguyen 2014; Amin 2017). 


\section{HIV, Biopolitik und die Sexualitätspolitik der Gegenwart}

Die Diagnose, Behandlung und Kontrolle von HIV und die dafür eingesetzten Gesundheitspolitiken haben direkte Auswirkungen auf schwule Sexualität und das schwule Leben. Foucaults Werk ist doppelt geeignet zur Analyse dieser Verhältnisse, denn sie sich erstens als Biopolitik beschreiben, die zweitens in sexuelle Subjektivierungen eingreift und sexuelle Ethiken und Praktiken verändert (Schubert 2019). Insbesondere werden mit Foucault neue Gouvernementalitäten der HIV-Prävention auf ihre normierenden und repressiven Effekte hin untersucht. In diesem Sinne beschreibt Tim Dean die Kultur des Kondomverzichts (bareback) als eine widerständige queere Praxis (Dean 2009) und die neue biomedizinische HIV-Prä-Expositions-Prophylaxe (PrEP) als ein Mittel zur gesteigerten Überwachung von schwulen Körpern (Dean 2015a; Orne/Gall 2019). Demgegenüber kann die Zusammenarbeit von medizinischer und aktivistischer Community bei der Etablierung von PrEP auch als »demokratische Biopolitik« verstanden werden (Schubert 2019). Die Bedeutung von PrEP für schwule Subjektivität liegt darin, dass es die seit den 1980ern aufgrund der AIDS-Krise vorherrschende Assoziation von schwulem Sex mit Tod und Krankheit beendet und so nicht nur zur Destigmatisierung beiträgt, sondern potentiell auch zur Überwindung von rigider Homonormativität, die Promiskuität auch wegen des Krankheitsstigmas ablehnt. Andere Beiträge zu PrEP finden sich in Varghese (2019). Einer Foucault'schen Normativitätskritik sind auch Analysen aktueller urbaner schwuler Sexkultur verpflichtet, wie der Benutzung von Online-Dating-Apps (Race 2015; Shield 2019) oder Chemsex, dem Gebrauch von Drogen beim Sex (Hakim 2018), die diese Kulturen und die damit verbundenen transformativen Ethiken jenseits eines pathologisierenden und sensationsgetriebenen Blicks darstellen. Solche Beiträge wehren sich auch gegen eine mit der Akademisierung einhergehende Desexualisierung der Queertheorie, die selbst ein Effekt von Homonormativität ist (Dean 2015b).

Die Aufgabe der Queertheorie - die Kritik an Hetero- und Homonormativität-ist aktueller denn je: Der aktuelle Rückschritt durch eine konservative Restauration, die in vielen Ländern schon zur Rückabwicklung von erkämpften Rechten führt, ist eine reale Gefahr für das Leben von queeren Personen. Die gesellschaftliche Basis für diese Homophobie wurde durch die assimilativen Strategien der Einforderung gleicher Rechte der vergangenen Jahre nicht erfolgreich bekämpft, wovon die queere Kritik dank ihres durch Foucault geschulten Blicks auf Macht jenseits des Rechts keineswegs überrascht ist. Unter der Oberfläche eines rechtlichen Fortschritts für privilegierte Schwule und Lesben herrscht nicht erst seit Trump und AfD ein»War 
on Sex«, deralle nicht-normativen Formen der Sexualität, insbesondere den kommerziellen, HIV-positiven und nicht-monogamen Sex bekämpft, wie im gleichnamigen Sammelband ausführlich analysiert wird (Halperin/Hoppe 2017). Ob dem konservativen Anti-Genderismus in Zukunft die Stirn geboten werden kann, entscheidet sich nicht zuletzt daran, ob es gelingt, queeres Denken in der Akademie voranzubringen.

\section{Literatur}

Amin, Kadji: Disturbing Attachments. Genet, Modern Pederasty, and Queer History. Durham 2017.

Berlant, Lauren/Warner, Michael: Sex in Public. In: Critical Inquiry 24/2 (1998), 547-566.

Berlant, Lauren Gail/Edelman, Lee: Sex, or the Unbearable. Durham 2014.

Bersani, Leo: Is the Rectum a Grave? In: October 43 (1987), AIDS: Cultural Analysis/Cultural Activism, 197-222.

Caserio, Robert L./Edelman, Lee/Halberstam, Judith/ Muñoz, José Esteban/Dean, Tim: The Antisocial Thesis in Queer Theory. In: PMLA 121/3 (2006), 819-828.

Dean, Tim: Unlimited Intimacy. Reflections on the Subculture of Barebacking. Chicago/London 2009.

Dean, Tim: Mediated Intimacies. Raw Sex, Truvada, and the Biopolitics of Chemoprophylaxis. In: Sexualities 18/1-2 (2015), 224-246. (2015a)

Dean, Tim: No Sex Please, We're American. In: Am Lit Hist 27/3 (2015), 614 624. (2015b)

Duggan, Lisa: The New Homonormativity. In: Russ Castronovo/Dana D. Nelson/Donald E. Pease/Joan Dayan/ Richard R. Flores (Hg.): Materializing Democracy. Durham 2002, 175-194.

Duggan, Lisa: Chapter 3: Equality, Inc. In: Lisa Duggan: The Twilight of Equality? Neoliberalism, Cultural Politics, and the Attack on Democracy. Boston MA 2003, 43-66.

Edelman, Lee: No Future. Queer Theory and the Death Drive. Durham/London 2004.

Eribon, Didier: Betrachtungen zur Schwulenfrage. Frankfurt a. M. 2019 (frz. 1999).

Foucault, Michel: Wahnsinn und Gesellschaft. Eine Geschichte des Wahns im Zeitalter der Vernunft. Frankfurt a. M. 1973 (frz. 1961).

Foucault, Michel: Der Wille zum Wissen. Sexualität und Wahrheit 1. Frankfurt a. M. 1983 (frz. 1976). 
Foucault, Michel: Von der Freundschaft als Lebensweise. Gespräch mit René de Ceccatty, Jean Danet und Jean Le Bitoux (Gai Pied). In: Michel Foucault: Von der Freundschaft als Lebensweise. Michel Foucault im Gespräch. Berlin 1984, 85-110.

Hakim, Jamie: The Rise of Chemsex. Queering Collective Intimacy in Neoliberal London. In: Cultural Studies 33/2 (2018), 249-275.

Halberstam, Judith: Trans*. A Quick and Quirky Account of Gender Variability. Oakland 2018.

Halperin, David M.: Saint Foucault. Towards a Gay Hagiography. New York 1995.

Halperin, David M.: How to Do the History of Male Homosexuality. In: GLQ: A Journal of Lesbian and Gay Studies 6/1 (2000), 87-123.

Halperin, David M.: The Normalization of Queer Theory. In: Journal of Homosexuality 45/2-4 (2003), 339-343. Halperin, David M.: How to be Gay. Cambridge, Mass. 2012. Halperin, David M./Hoppe, Trevor (Hg.): The War on Sex. Durham 2017.

Halperin, David M./Traub, Valerie (Hg.): Gay Shame. Chicago 2009.

Huffer, Lynne: Mad for Foucault. Rethinking the Foundations of Queer Theory. New York u. a. 2010.

Keegan, Cáel M.: Getting Disciplined. What's Trans* About Queer Studies Now? In: Journal of Homosexuality (2018), 1-14.

Laufenberg, Mike: Sexualität und Biomacht. Vom Sicherheitsdispositiv zur Politik der Sorge. Bielefeld 2014.

McWhorter, Ladelle: Bodies and Pleasures. Foucault and the Politics of Sexual Normalization. Bloomington 1999.

Muñoz, José Esteban: Cruising Utopia. The Then and There of Queer Futurity. New York/London 2009.

Nguyen, Hoang Tan: A View from the Bottom. Asian American Masculinity and Sexual Representation. Durham 2014.

Orne, Jason/Gall, James: Converting, Monitoring, and Policing PrEP Citizenship. Biosexual Citizenship and the PrEP Surveillance Regime. In: Surveillance \& Society 17/5 (2019), 641-661.

Preciado, Beatriz: Testo Junkie. Sex, Drugs, and Biopolitics in the Pharmacopornographic Era. Übers. von Bruce Benderson. New York 2013.

Puar, Jasbir K.: Terrorist Assemblages. Homonationalism in Queer Times. Durham 2007.

Race, Kane: >Party and Playı. Online Hook-Up Devices and the Emergence of PNP Practices among Gay Men. In: Sexualities 18/3 (2015), 253-275.

Rubin, Gayle S.: Thinking Sex [1986]. In: Gayle S. Rubin: Deviations: A Gayle Rubin Reader. Durham/London 2011. 
Schubert, Karsten: Langer Weg zur sexuellen Selbstbestimmung. Der Schutz von LSBTI durch die Vereinten Nationen. In: Vereinte Nationen 61/5 (2013), 216-222.

Schubert, Karsten: The Democratic Biopolitics of PrEP. In: Helene Gerhards/Kathrin Braun (Hg.): Biopolitiken - Regierungen des Lebens heute. Wiesbaden 2019, 121-153.

Scott, Darieck: Extravagant Abjection. Blackness, Power, and Sexuality in the African American Literary Imagination. New York 2010.

Sedgwick, Eva Kosofsky: Epistemology of the Closet. Berkeley 1990.

Shield, Andrew D. J.: Immigrants on Grindr. Race, Sexuality and Belonging Online. London 2019.

Spargo, Tamsin: Foucault and Queer Theory. Duxford 1999. Spector, Scott/Puff, Helmut/Herzog, Dagmar (Hg.): After the

History of Sexuality. German Genealogies with and beyond Foucault. New York 2012.

Trott, Ben (2016): Same-Sex Marriage and the Queer Politics of Dissensus. In: South Atlantic Quarterly 115/2 (2016), 411-423.

Varghese, Ricky (Hg.): Raw. Prep, Pedagogy, and the Politics of Barebacking. London 2019.

Warner, Michael: Fear of a Queer Planet. Queer Politics and Social Theory. Minneapolis/London 1993.

Warner, Michael: The Trouble with Normal. Sex, Politics, and the Ethics of Queer Life. Cambridge, Mass. 2000. 